\title{
Discovery of Potent, Selective, and Orally Bioavailable Inhibitors of USP7 with In Vivo Antitumor Activity
}

Paul R. Leger ${ }^{\ddagger}$, Dennis X. Hu ${ }^{\ddagger}$, Berenger Biannic ${ }^{\ddagger}$, Minna Bui ${ }^{\ddagger}$, Xinping Han ${ }^{\ddagger}$, Emily Karbarz ${ }^{\ddagger}$, Jack Maung ${ }^{\ddagger}$, Akinori Okano ${ }^{\ddagger}$, Maksim Osipov ${ }^{\ddagger}$, Grant M. Shibuya ${ }^{\ddagger}$, Kyle Young ${ }^{\ddagger}$, Christopher Higgs $^{\S}$, Betty Abraham ${ }^{\ddagger}$, Delia Bradford ${ }^{\ddagger}$, Cynthia Cho ${ }^{\ddagger}$, Christophe Colas $\$$, Scott Jacobson Yamini M. Ohol ${ }^{\ddagger}$, Deepa Pookot ${ }^{\ddagger}$, Payal Rana ${ }^{\ddagger}$, Jerick Sanchez ${ }^{\ddagger}$, Niket Shah ${ }^{\ddagger}$, Michael Sun ${ }^{\ddagger}$, Steve Wong ${ }^{\ddagger}$, Dirk G. Brockstedt ${ }^{\ddagger}$, Paul D. Kassner ${ }^{\ddagger}$ Jacob B. Schwarz ${ }^{\ddagger}$, David J. Wustrow*

RAPT Therapeutics, 561 Eccles Avenue, South San Francisco, CA 94080

${ }^{\S}$ Schrödinger, 120 West $45^{\text {th }}$ Street, New York, NY 10036

\section{Supporting Information}

Table of Contents:

Biochemical and cellular potency correlation................... Page S2

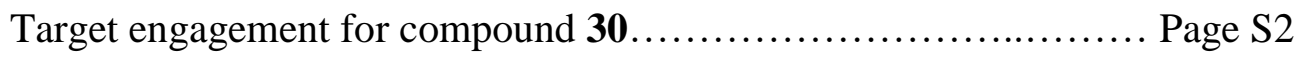

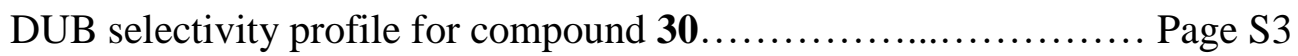

View of USP7 showing solvent-exposed Tyr-465 ............... Page S3

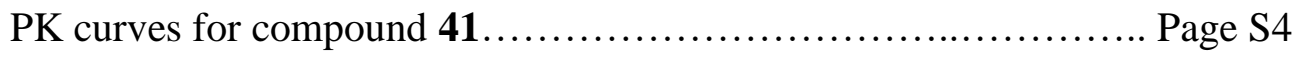




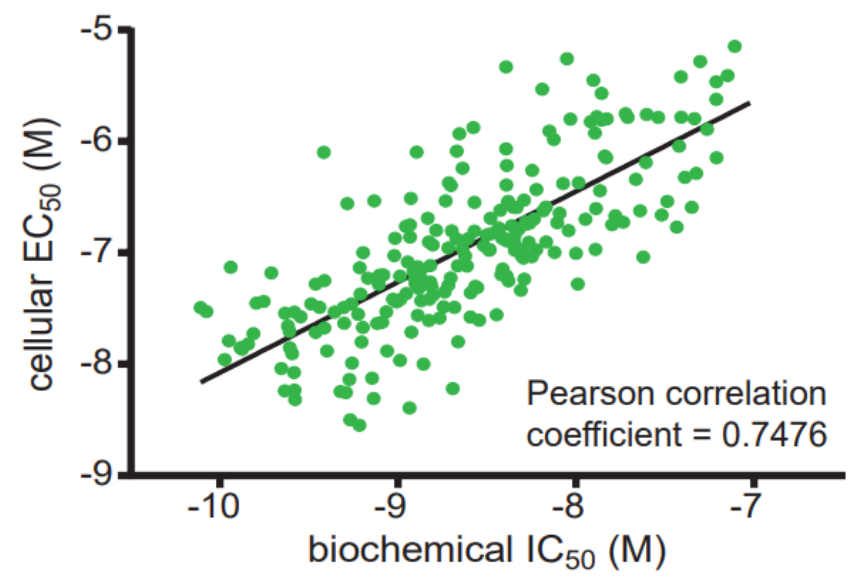

Figure S1. Correlation between the USP7 biochemical $\mathrm{IC}_{50} \mathrm{~S}$ and the cellular p53 $\mathrm{EC}_{50} \mathrm{~S}$ for the USP7 inhibitors of the chemotypes outlined in this paper.

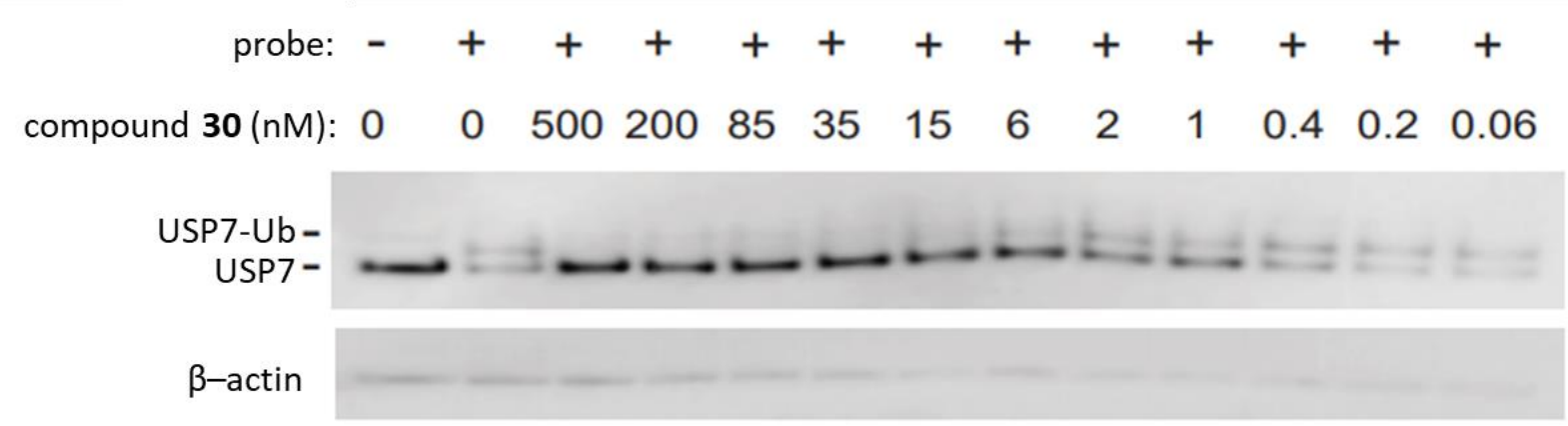

Figure S2. USP7 target engagement by compound $\mathbf{3 0}$ is demonstrated using the activity-based Ub-PA probe. When compound $\mathbf{3 0}$ is added, a dose-proportional decrease in USP7-Ub and an increase in USP7 is observed, demonstrating that $\mathbf{3 0}$ can engage USP7 and prevent its binding to and conjugation with the active-site probe. Cells were seeded at 1 million per well in recommended media and treated for 4 hours with compound 30. Cells were lysed in IP Lysis Buffer (Pierce) and lysates were quantified using the BCA assay (Pierce). $20 \mu \mathrm{g}$ lysate was treated with $2 \mu \mathrm{M}$ Ubiquitinpropargylamide (Ub-PA) probe (UbiQ-057) for 15 minutes at room temperature, following which probe activity was stopped by the addition of $1 \times$ LDS Sample Buffer (ThermoFisher). Samples were heated at $70^{\circ} \mathrm{C}$ for 10 minutes, separated by SDSPAGE, and analyzed by Western blotting using anti-USP7 (Millipore 05-1946, 1:2000), anti- $\beta$-actin (Invitrogen MA515739, 1:5000), and goat anti-mouse HRP (Invitrogen 31430, 1:5000) antibodies. 


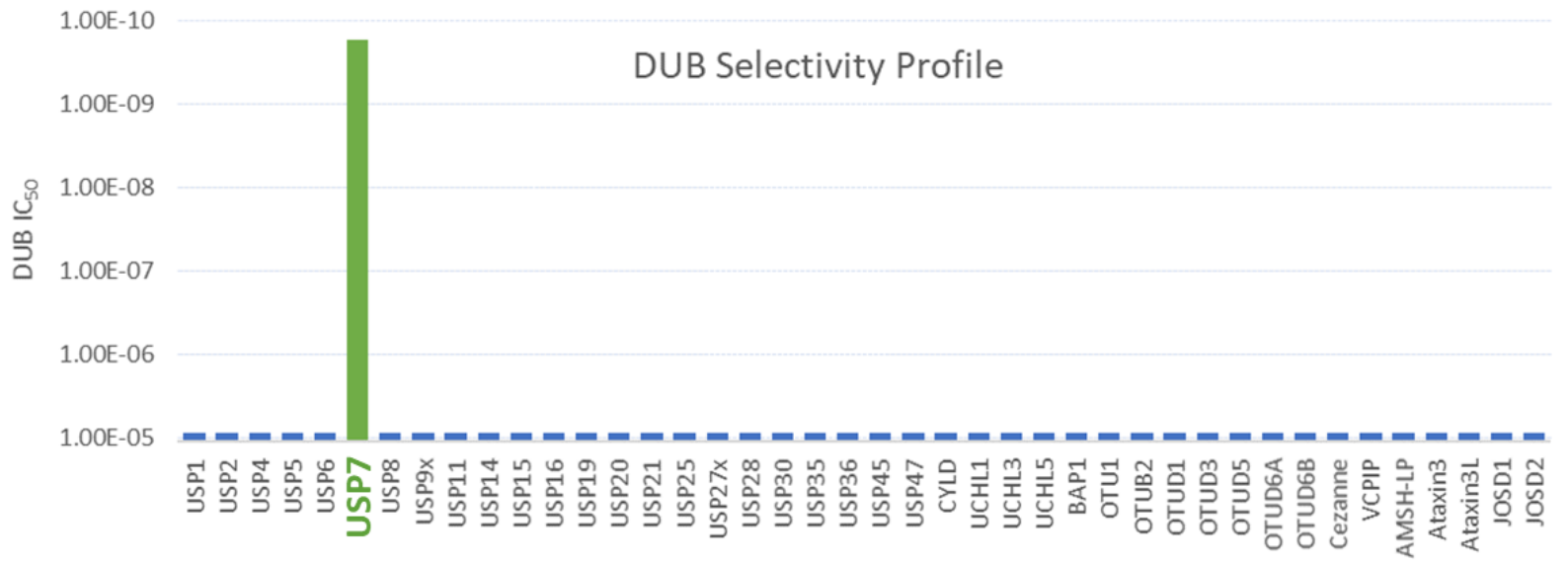

Figure S3. Compound 30 was profiled for DUB selectivity at Ubiquigent (Dundee, UK). Compound $\mathbf{3 0}$ was assayed for biochemical inhibition against a wide range of DUBs shown in the figure. Compound 30 showed an $\mathrm{IC}_{50}>10 \mu \mathrm{M}$ for all the DUBs that were assayed (except USP7), corresponding to a >50,000-fold selectivity for USP7 over the other DUBs.

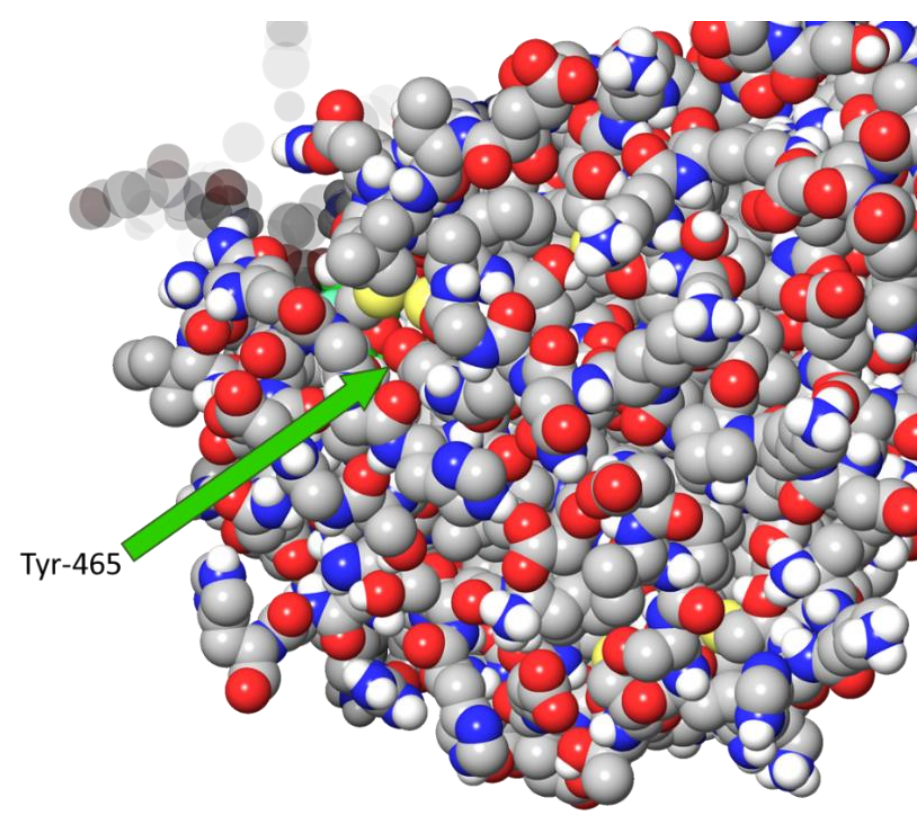

Figure S4. The oxygen atom of Tyr-465 (highlighted by green arrow) is solvent-exposed through a small tunnel (opposite the ligand binding site) in the USP7 protein. The figure demonstrates this utilizing the co-crystal structure of compound 23 with USP7, PDB code 6VN3. 

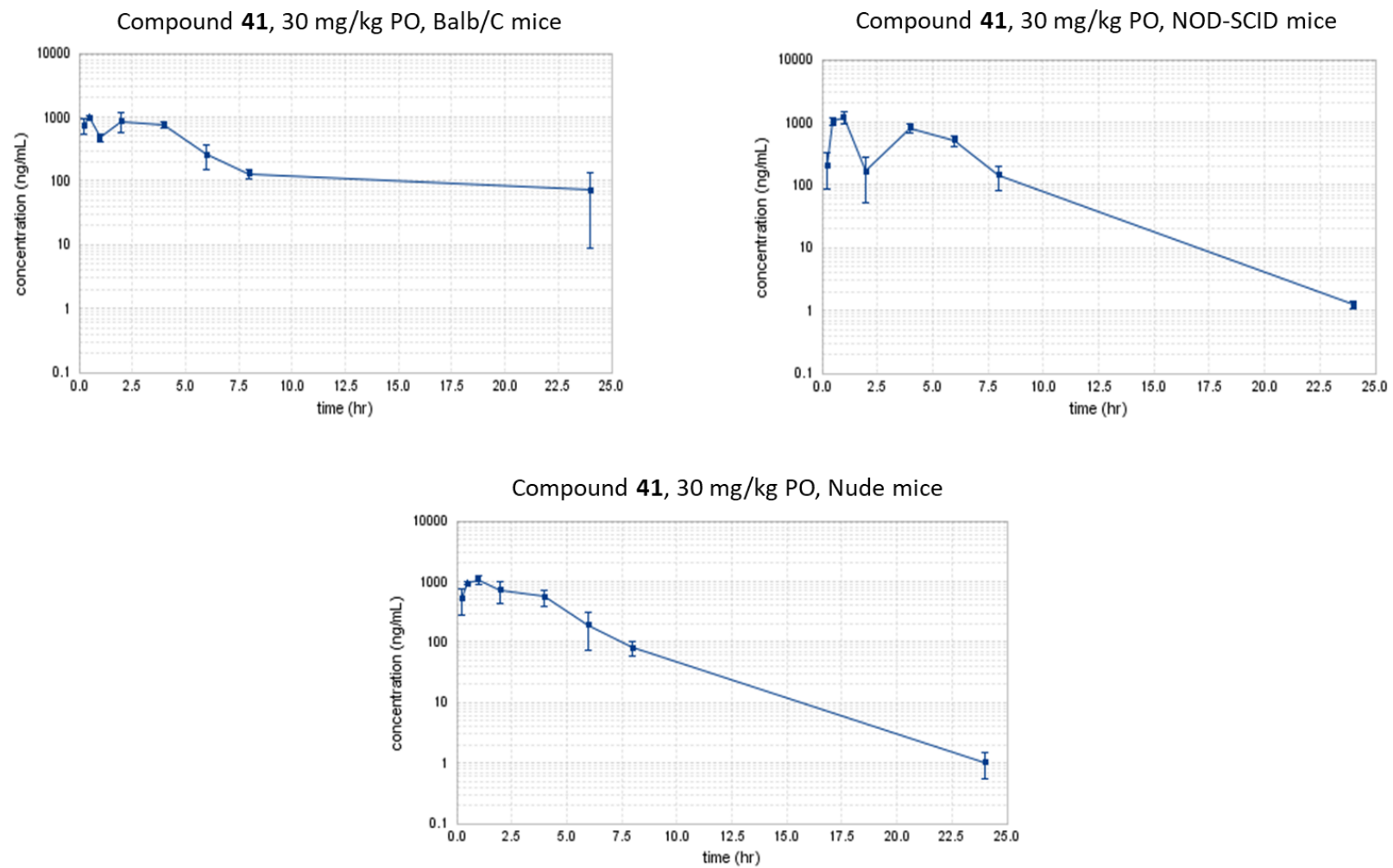

Figure S5. PK curves for a $30 \mathrm{mg} / \mathrm{kg}$ PO dose of compound 41 in Balb/C mice, NOD-SCID mice, or Nude mice. Each data point is the average of $n=3$ animals. 